\section{Calidad en cirugía: hacia una mejor comprensión de las complicaciones quirúrgicas}

\author{
RICARDO ESPINOZA G., JUAN PABLO ESPINOZA G.
}

\section{Quality assessment in surgery}

This paper deals with quality from the perspective of structure, processes and indicators in surgery. In this specialty, there is a close relationship between effectiveness and quality. We review the definition and classification of surgical complications as an objective means of assessing quality. The great diversity of definitions and risk assessments of surgical complications hampered the comparisons of different surgical centers or the evaluation of a single center along time. We discuss the different factors associated with surgical risk and some of the predictive systems for complications and mortality. At the present time, standarized definitions and comparisons are carried out correcting for risk factors. Thus, indicators of mortality, complications, hospitalization length, postoperative quality of life and costs become comparable between different groups. The volume of procedures of a determinate center or surgeon as a quality indicator is emphasized.

(Rev Med Chile 2016; 144: 757-765)

Key words: Hospital Mortality; Morbidity; Mortality; Operative Procedures; Outcomes Assessment (Health Care); Patient Outcome Assessment; Surgical Procedures, Operative.

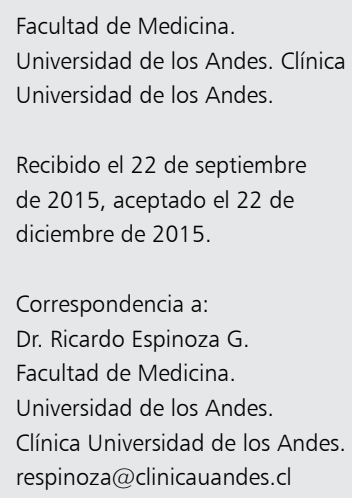

$\mathrm{D}$ entro de las seis dimensiones que comprende la Calidad en Medicina, a saber: seguridad, oportunidad, eficiencia, y que sea efectiva, equitativa y centrada en el paciente ${ }^{1}$, la efectividad y seguridad son aquellas dos que más directamente se asocian con los tratamientos quirúrgicos. Pareciera ser que en la Cirugía se hace más evidente la relación entre tratamiento efectuado y los resultados que se siguen y la superioridad que tienen éstos frente a otras alternativas terapéuticas.

Hace ya bastantes años, Donabedian ${ }^{2}$ analizó la Calidad en Cirugía y determinó tres ámbitos: estructura, procesos y resultados. En el primer grupo están contenidos los profesionales con sus grados de especialización y, por otro lado, los recursos físicos de cada centro. Cuando se habla de procesos en la atención, se entiende que ellos deben ser completos y deben estar basados en la mejor evidencia. Los resultados, aquellas mediciones que tradicionalmente se utilizan para medir calidad son variados, y entre ellos podemos mencionar: mortalidad, morbilidad, días de hospitalización, costos, calidad de vida postoperatoria y sobrevida a largo plazo, entre otros (Tabla 1).

En este artículo analizaremos con especial atención la morbilidad quirúrgica, para entender mejor la multiplicidad de elementos que, al intervenir quirúrgicamente a un paciente, están relacionados a la morbilidad. Estos elementos han estado siendo motivo de extensa investigación y revisión, pues los resultados quirúrgicos interesan por cierto a los pacientes, a los mismos médicos, a los administradores hospitalarios y a los aseguradores $^{3,4}$. Las complicaciones comportan dolor y costos; creemos que el mejor entendimiento de ellas, orientado a la prevención, se puede traducir en una disminución de la morbilidad 5 . 
Tabla 1 Calidad en cirugía

\begin{tabular}{|lll|}
\hline Estructura & Resultados & Procesos \\
Recurso humano: Número y grado de especialización, & Morbilidad postoperatoria & Adecuados y oportunos \\
educación médica continua & Mortalidad postoperatoria & Completos \\
Recurso físico: Infraestructura y equipamiento & Sobrevida & Basados en la Evidencia \\
& Calidad de vida postoperatoria & \\
& Días de hospitalización & \\
& Costos \\
& Satisfacción del paciente \\
\hline
\end{tabular}

\section{Morbilidad postoperatoria}

De forma reiterada estamos comparando los resultados quirúrgicos. Pero es notable que frente a ellos hemos convivido con varios problemas. Hasta hace poco, no ha habido acuerdo sobre las definiciones y no se entendía del mismo modo lo que significan las complicaciones. La mejor definición de morbilidad postoperatoria sería la de aquella desviación del curso postoperatorio normal; esto implica conocer la normalidad de una evolución para estar atento a detectar estas desviaciones. De forma complementaria, podemos entender la morbilidad postoperatoria como todos aquellos eventos adversos que ocurren con ocasión de una cirugía. Tradicionalmente se siguen analizando las complicaciones hasta los 30 días siguientes a una intervención; sin embargo, ya bastantes grupos han prolongado este plazo hasta los 100 días $^{6}$.

Una segunda dificultad que ha hecho difícil las comparaciones, es la definición propiamente tal de cada complicación específica. Martin ${ }^{7}$ dio algunos ejemplos, observando en la literatura cinco maneras diferentes de definir una fístula biliar post hepatectomía y 12 definiciones distintas para fístula pancreática; obviamente que así es imposible comparar resultados. Una tercera dificultad queda reflejada por la forma de notificación de las complicaciones. Es comprensible que cuando la notificación queda librada a la sola voluntad e iniciativa, las tasas reportadas sean bajas. La experiencia en el Massachusetts General Hospital demostró que con la implementación de un programa específico de notificación, la morbilidad y mortalidad de los servicios de cirugía crecía sustancialmente, no por una mayor incidencia, sino por el real conocimiento de su frecuencia ${ }^{8,9}$.
Cuando se conocían las complicaciones y muertes sólo por medio de las reuniones de Morbilidad y Mortalidad, éstas eran de $6,4 \%$ y $0,9 \%$ y con la aplicación de un programa específico, de $28,9 \%$ y $1,9 \%$, respectivamente.

Por último, otro elemento importante ha sido catalogar y así poder comparar, la gravedad de las complicaciones, pues entendemos que hay complicaciones más graves que otras. Desde hace ya dos décadas se han realizado intentos muy completos para poder tener una graduación ascendente, entendiendo que la complicación más grave es la muerte del paciente ${ }^{10}$. El primer intento se lo debemos a Clavien ${ }^{11}$, quien en 1992 propuso una estratificación de la gravedad de las complicaciones, clasificación que luego fue mejorada por Dindo, dando lugar a la clasificación de Clavien-Dindo del año 2004 ${ }^{12,13}$. En el intertanto, Martin $^{7}$, del Memorial Sloan-Kettering Cancer Center de Nueva York también propuso una clasificación por gravedad. Más recientemente, Strasberg en el 2009 propuso el sistema Accordion ${ }^{14}$. Estos sistemas de clasificación de la gravedad de las complicaciones se basan en el tratamiento que estas complicaciones demandan. Así, aquellas complicaciones leves se resuelven en la misma cama del enfermo y requieren procedimientos invasivos menores, algunos catéteres o sondas, diuréticos y antieméticos entre otros. Las complicaciones de gravedad moderada requieren antibióticos, transfusiones o apoyo nutricional parenteral. En cambio, las complicaciones graves conllevan una reintervención quirúrgica o algún procedimiento invasivo radiológico o endoscópico. Dijimos que la muerte del paciente representa la complicación más grave (Tabla 2). La clasificación de Clavien y Accordion son las más utilizadas en la práctica clínica. 
Tabla 2. Clasificación Accordion (Abreviada) de gravedad de las complicaciones postoperatorias

1. Complicaciones leves: Requieren sólo procedimientos invasivos menores que se pueden realizar al lado de la cama del paciente, tales como la inserción de una vía intravenosa, un catéter urinario, tubo nasogástrico y drenaje de infecciones de herida. Fisioterapia y las siguientes drogas son permitidas: antieméticos, antipiréticos, analgésicos, diuréticos y electrolitos

2. Complicaciones moderadas: Requieren tratamiento farmacológico con drogas distintas a las permitidas para las complicaciones menores, por ejemplo antibióticos. Transfusiones sanguíneas y nutrición parenteral total están incluidas

3. Complicaciones graves: Todas las complicaciones que requieren procedimientos endoscópicos o radiológicos intervencionales o requieren reoperación. También las complicaciones que resultan en la falla de uno o más órganos/sistemas

\section{Muerte postoperatoria}

Tabla 3. Factores predictivos de morbilidad postoperatoria

Disminución de la albúmina sérica $(\mathrm{g} / \mathrm{dl})$
Clasificación $\mathrm{ASA}^{*}$
Complejidad de la cirugía
Cirugía de urgencia
Edad
Estado funcional (independencia/dependencia)
Hematocrito $\leq 38 \%$
Leucocitos $>11.000 / \mathrm{mm}^{3}$
Plaquetas $\leq 150.000 / \mathrm{mm}^{3}$
Baja de peso $>10 \%$ en 6 meses
Historia de enfermedad pulmonar obstructiva crónica
BUN $>40$ mg/dl
Ventilación mecánica
*ASA: American Society of Anesthesiology.

*ASA: American Society of Anesthesiology.

la historia respiratoria y signos de falla cardíaca y la catalogación en la Escala de Coma de Glasgow, incluyó varias mediciones de laboratorio, sumando doce parámetros relacionados con las complicaciones (Tabla 4).

En Japón, en el año 1999, Haga ${ }^{18}$ identificó en el preoperatorio seis factores clínicos de riesgo: edad, enfermedad cardíaca, enfermedad pulmonar, diabetes mellitus, clasificación ASA y estado de dependencia/independencia del paciente, que juntos conforman el Preoperative Risk Score (Tabla 5).

Conocidos estos factores de riesgo se trabajó en producir modelos de ajuste de riesgo para conocer el nivel preoperatorio de enfermedad y así poder comparar resultados con el riesgo ajustado según las características previas de los pacientes; es decir, ajustados a cuán enfermos estaban al momento de ser tratados quirúrgicamente. Primero se adquirió información prospectiva y luego se construyeron los modelos. En 1994, a nivel de VHA ${ }^{16}$ se estableció el National Surgical Quality Improvement Program (NSQIP) con lo que se contó finalmente con una herramienta válida para seguir en el tiempo, comparar y mejorar los resultados quirúrgicos ajustados a los factores de riesgo. 
Tabla 4. Valoración fisiológica preoperatoria. Modelo POSSUM

\begin{tabular}{|c|c|c|c|c|}
\hline & 1 & 2 & 3 & 8 \\
\hline Edad (años) & $\leq 60$ & $61-70$ & $\geq 71$ & \\
\hline Signos cardiacos & Sin falla & $\begin{array}{l}\text { Terapia con diuréticos, } \\
\text { digoxina, antianginosos } \\
\text { o antihipertensivos }\end{array}$ & $\begin{array}{c}\text { Edema periférico. } \\
\text { Terapia con warfarina. } \\
\text { Cardiomegalia límite (Rx) }\end{array}$ & $\begin{array}{c}\text { Presión venosa yugular } \\
\text { elevada. } \\
\text { Cardiomegalia (Rx) }\end{array}$ \\
\hline Historia respiratoria & Sin disnea & Disnea ejercicio & Disnea limitante & $\begin{array}{c}\text { Disnea de reposo } \\
\text { (FrecResp } \geq 30 / \mathrm{min} \text { ) }\end{array}$ \\
\hline $\begin{array}{l}\text { Presión sanguínea } \\
\text { sistólica }(\mathrm{mmHg})\end{array}$ & $110-130$ & $\begin{array}{l}131-170 \\
100-109\end{array}$ & $\begin{array}{l}\geq 171 \\
90-99\end{array}$ & $\leq 89$ \\
\hline Pulso (latidos/min) & $50-80$ & $\begin{array}{c}81-100 \\
40-49\end{array}$ & $101-120$ & $\begin{array}{l}\geq 121 \\
\leq 39\end{array}$ \\
\hline $\begin{array}{l}\text { Escala de coma } \\
\text { de Glasgow }\end{array}$ & 15 & $12-14$ & $9-11$ & $\leq 8$ \\
\hline $\begin{array}{l}\text { Hemoglobina } \\
(\mathrm{g} / 100 \mathrm{ml})\end{array}$ & $13-16$ & $\begin{array}{l}11,5-12,9 \\
16,1-17,0\end{array}$ & $\begin{array}{l}10,0-11,4 \\
17,1-18,0\end{array}$ & $\begin{array}{r}\leq 9,9 \\
\geq 18,1\end{array}$ \\
\hline Leucocitos (x1012/I) & $4-10$ & $\begin{array}{l}10,1-20 \\
3,1-4,0\end{array}$ & $\begin{array}{l}\geq 20,1 \\
\leq 3,0\end{array}$ & \\
\hline Urea (nmol/l) & $\leq 7,5$ & $7,6-10,0$ & $10,1-15,0$ & $\geq 15,1$ \\
\hline Sodio (nmol/l) & $\geq 136$ & $131-135$ & $126-130$ & $\leq 125$ \\
\hline Potasio (nmol/l) & $3,5-5,0$ & $\begin{array}{l}3,2-3,4 \\
5,1-5,3\end{array}$ & $\begin{array}{l}2,9-3,1 \\
5,4-5,9\end{array}$ & $\begin{array}{l}\leq 2,8 \\
\geq 6,0\end{array}$ \\
\hline Electrocardiograma & Normal & & $\begin{array}{c}\text { Fibrilación auricular } \\
\text { (frecuencia 60-90) }\end{array}$ & $\begin{array}{c}\text { Otro ritmo anormal, } \geq 5 \\
\text { ectópicos/min, ondas Q } \\
\text { o cambios en el ST/T }\end{array}$ \\
\hline
\end{tabular}

Tabla 5. Factores de Riesgo Preoperatorio. Modelo E-PASS (Estimation of Physiologic Ability and Surgical Stress)

\begin{tabular}{|l|}
\hline Edad \\
\hline Enfermedad cardíaca \\
\hline Enfermedad pulmonar \\
\hline Diabetes mellitus \\
Clasificación ASA* \\
Estado de independencia/dependencia \\
\hline
\end{tabular}

*ASA: American Society of Anesthesiology.

\section{Sistemas predictivos de morbilidad y mortalidad postoperatoria}

A los factores de riesgo reconocidos por Copeland ${ }^{17}$, le siguió la estructuración de un sistema que incorporó diversos parámetros operatorios: gravedad de la cirugía, si se trataba de un procedimiento único o múltiple, las pérdidas sanguíneas, el grado de contaminación peritoneal, la presencia de cáncer y si la cirugía era de urgencia o electiva. Con todos esos elementos se construyó el modelo predictivo de morbimortalidad POSSUM que ha sido aplicado en múltiples estudios quirúrgi$\cos ^{19-21}$.

$\mathrm{Haga}^{18}$, entendiendo que las complicaciones derivan de factores de riesgo y el estrés quirúrgico, construyó el Surgical Stress Score ${ }^{22}$ que contempla pérdidas sanguíneas, tiempo operatorio y extensión de la incisión, que combinado con los factores de riesgo por él identificados, dio origen al Comprehensive Risk Score que permite en el preoperatorio hacer un cálculo individual para estimar el riesgo de complicaciones y mortalidad de un paciente específico frente a una cirugía determinada. Es el sistema conocido como E-PASS (Estimation of Physiologic Ability and Surgical 
Stress) que puede ayudar a planificar un tratamiento quirúrgico ${ }^{23,24}$. No obstante, ni el sistema de Copeland ni el de Haga han penetrado en la práctica clínica habitual.

El Colegio Americano de Cirujanos (American College of Surgeons, ACS) desde el año 2005 expandió el Programa de Mejoramiento de la Calidad en Cirugía (National Surgical Quality Improvement Program, NSQIP) iniciado por VHA, incorporando hospitales privados ${ }^{25}$. Con el ACS se ha construido un programa nacional validado, basado en resultados y ajustado por riesgo, colocando a disposición una herramienta de cálculo del riesgo quirúrgico para un paciente particular frente a una cirugía determinada. Considera 21 predictores que, asociados a un procedimiento definido, puede predecir nueve posibles evoluciones dentro de los primeros 30 días de la cirugía. Es materia de recientes publicaciones ${ }^{26,27}$. En la actualidad, los ajustes y comparaciones en calidad se hacen fundamentalmente sobre las observaciones del NSQIP, en hospitales de Norteamérica.

\section{Factor volumen hospital y volumen cirujano}

En el año 1957, Lee dio a conocer en Lancet ${ }^{28}$ una primera observación sobre el impacto que tenía el volumen de un hospital sobre la evolución de sus pacientes quirúrgicos. Las observaciones fueron refrendadas por Luft en $1979^{29}$ y desde entonces han sido innumerables las comunicaciones que han puesto en evidencia la relación entre volumen quirúrgico de un hospital y los resultados. El grupo de Birkmeyer ${ }^{30-32}$ ha sido el que más ha ilustrado este asunto. En New England Journal of Medicine 2002, este autor ${ }^{33}$ dio a conocer los resultados en ciertas cirugías cardiovasculares (endarterectomía carotídea, puentes arteriales en extremidades inferiores, reemplazo aórtico abdominal electivo, revascularización coronaria, reemplazo aórtico y reemplazo mitrálico) y ciertas cirugías oncológicas tales como colectomía, gastrectomía, resección pancreática, nefrectomía, cistectomía, lobectomía pulmonar y neumonectomía), relacionando el número de intervenciones con la mortalidad. Le siguieron otros trabajos que mostraron resultados similares, esto es: a mayor volumen hospitalario, menor morbimortalidad, y con ello, se comenzó a hablar de centros de alto volumen (CAV) y centros de bajo volumen (CBV). En base a este conocimiento, y en conjunto con grandes aseguradoras norteamericanas, surgió la idea de regionalización y derivación de ciertos pacientes a los CAV por sus mejores resultados. Fue el mismo Birkmeyer ${ }^{31}$ quien estimó en base a estas observaciones, las vidas potencialmente salvadas si los pacientes eran operados en CAV (Tabla 6), y se comenzó a hablar del concepto de regionalización ${ }^{34}$. No obstante, también se reconocieron las enormes dificultades para ampliar una iniciativa de este tipo y los esfuerzos se dirigieron más bien a identificar los factores determinantes de esos mejores resultados, para poder traspasarlos e implementarlos en los otros centros, donde se observaban mayores cifras de morbimortalidad.Otros autores ${ }^{32,35,36}$ confirmaron las observaciones iniciales y observaron que en patología oncológica, si la cirugía era efectuada en CAV se obtenían hospitalizaciones más breves, una mortalidad inferior, mayor sobrevida a 5 años y menores tasas de recurrencia. Sin embargo, no era muy claro el límite de definición que permitía categóricamente distinguir un CAV de un CBV. Además, en los CAV tendían a trabajar los cirujanos más especializados, los casos quirúrgicos

Tabla 6. Mortalidad proyectada a 30 días (\%) en Hospitales de Alto Volumen (HAV) y Hospitales de Bajo Volumen (HVB). Vidas Potencialmente Salvadas (VPS)

\begin{tabular}{|lccc|}
\hline Tipo cirugía & Mortalidad HAV (\%) & Mortalidad HBV (\%) & VPS (n) \\
\hline Revascularización coronaria & 2,4 & 3,3 & 1.486 \\
\hline Angioplastía coronaria & 0,9 & 1,2 & 345 \\
\hline Reparación electiva de AAA & 4,2 & 6,7 & 464 \\
\hline Endarterectomía carotídea & 0,5 & 0,7 & 118 \\
\hline Esofagectomía & 5,9 & 15,8 & 168 \\
\hline
\end{tabular}

*AAA: Aneurisma aorta abdominal. 
Tabla 7. Mortalidad Quirúrgica a 30 días (\%)

\begin{tabular}{|c|c|c|c|c|}
\hline & \multicolumn{2}{|c|}{ Mortalidad/volumen hospital } & \multicolumn{2}{|c|}{ Mortalidad/volumen cirujano } \\
\hline & Más alto & Más bajo & Más alto & Más bajo \\
\hline \multicolumn{5}{|l|}{ Cirugías cardiovasculares } \\
\hline Endarterectomía carotídea & 1,5 & 1,7 & 1,1 & 1,8 \\
\hline Puente vascular extremidad inferior & 4,1 & 5,1 & - & - \\
\hline Reparación electiva $A A^{*} *$ & 3,9 & 6,5 & 3,9 & 6,2 \\
\hline Revascularización coronaria & 4,5 & 5,6 & 4,0 & 5,4 \\
\hline Reemplazo valvular aórtico & 7,1 & 9,3 & 6,5 & 9,1 \\
\hline Reemplazo valvular mitral & 11,6 & 15,1 & - & - \\
\hline \multicolumn{5}{|l|}{ Cirugías oncológicas } \\
\hline Colectomía & 4,5 & 5,6 & - & - \\
\hline Gastrectomía & 8,6 & 11,4 & - & - \\
\hline Esofagectomía & 8,4 & 20,3 & 9,2 & 18,8 \\
\hline Pancreatectomía & 3,8 & 16,3 & 4,6 & 14,7 \\
\hline Nefrectomía & 2,1 & 2,6 & 3,1 & 5,5 \\
\hline Cistectomía & 2,6 & 5,5 & - & - \\
\hline Lobectomía pulmonar & 4,0 & 5,7 & - & - \\
\hline Neumonectomía & 10,7 & 16,1 & - & - \\
\hline
\end{tabular}

*AAA: aneurisma aorta abdominal.

eran obviamente más frecuentes, existían mejores unidades de cuidados críticos y más especialistas de apoyo y en general, estaban mejor definidos los procesos que definían los cuidados postoperatorios. Comenzó, por tanto, a plantearse cuánto de los mejores resultados eran por el sólo hecho de tratarse de un CAV y cuánto se debía al volumen del cirujano propiamente tal. El mismo Birkmeyer en el $2003^{30}$ comunicó el efecto volumen del cirujano sobre los resultados quirúrgicos y este mismo efecto en pacientes sometidos a cirugía oncológica fue demostrado por Brennan ${ }^{37}$ (Tablas 7 y 8 ). Con todo ello se estableció un mapa en que se han identificado cirugías más dependientes del volumen del cirujano y otras que son más dependientes del volumen del hospital. A saber, un reemplazo valvular aórtico, la reparación de un aneurisma de la aorta abdominal, una resección de páncreas y de esófago son más dependientes del volumen del cirujano y para las resecciones pulmonares, cistectomías y resecciones hepáticas es relativamente mayor el peso del volumen del hospital. Pero, como fue insinuado, ambos efectos, el volumen del hospital y el volumen del cirujano no son completamente separables y los dos están relacionados.
Tabla 8. Mortalidad en cirugía oncológica a 30 días (\%) de acuerdo a volumen/hospital y volumen/cirujano

\begin{tabular}{|ll|ccc|}
\hline & & \multicolumn{3}{c|}{ Volumen cirujano } \\
\hline \multirow{2}{*}{$\begin{array}{l}\text { Volumen } \\
\text { hospital }\end{array}$} & Alto & 1,36 & 1,87 & 2,57 \\
& Mediano & 1,61 & 2,04 & 2,72 \\
\cline { 2 - 5 } & Bajo & 2,81 & 3,02 & 3,48 \\
\hline
\end{tabular}

En el 2009, Nathan, del Hospital Johns Hopkins $^{38}$, estudió dirigidamente el volumen hospital y volumen cirujano sobre dos tipos de intervenciones, cirugía resectiva hepática y resecciones pancreáticas, notando que para la cirugía pancreática es muy significativo el volumen operatorio del cirujano, como también es importante para la cirugía hepática; pero para esta última, es más significativo contar con un buen hospital que proporcione cuidados perioperatorios de excelencia, con apoyo a nivel de cuidados críticos y de otros especialistas, como radiólogos intervencionales.

Estudios posteriores, ${ }^{5,39-41}$, analizando nuevamente las cirugías índice, hicieron ver que la mor- 
Tabla 9. Mortalidad, complicaciones y fallas en el rescate en los "mejores " y "peores" hospitales (\%)

\begin{tabular}{|c|c|c|c|c|c|c|}
\hline \multirow[b]{2}{*}{ Tipo cirugía } & \multicolumn{2}{|c|}{ Mortalidad } & \multicolumn{2}{|c|}{ Complicaciones } & \multicolumn{2}{|c|}{ Fallas en el rescate } \\
\hline & "Mejores" & "Peores" & "Mejores" & "Peores" & "Mejores" & "Peores" \\
\hline Revascularización coronaria & 2,6 & 4,7 & 21,1 & 24,2 & 6,2 & 18,9 \\
\hline Reparación electiva AAA * & 2,1 & 3,8 & 14 & 20,8 & 3,4 & 33,8 \\
\hline Reemplazo valvular aórtico & 4,1 & 7,5 & 27,1 & 33,5 & 7,2 & 26 \\
\hline Reemplazo valvular mitral & 7,4 & 12,8 & 36,3 & 46,2 & 6,6 & 43,1 \\
\hline Pancreatectomía & 2,8 & 9,1 & 22,4 & 40,8 & 4 & 50,8 \\
\hline Esofagectomía & 6,3 & 12,1 & 29,4 & 48,1 & 5,7 & 56,9 \\
\hline
\end{tabular}

*AAA: aneurisma aorta abdominal.

talidad en los "peores hospitales" era efectivamente 2,5 veces mayor que en los "mejores hospitales", pero que las diferencias en las complicaciones no eran tan marcadas como en la mortalidad; lo que hacía la diferencia entre unos hospitales y otros era la capacidad que cada hospital tenía para sacar adelante a sus pacientes complicados. Es decir, lo que era diferentes era la capacidad de "rescate de complicaciones" entre unos y otros (Tabla 9). Un estudio publicado en $2013^{42}$ del Michigan Surgical Quality Collaborative demostró que en los hospitales de alta mortalidad, existía una falla de $42,1 \%$ para rescatar de las complicaciones, a diferencia de $27,4 \%$ de falla que tenían los hospitales con baja mortalidad para sacar adelante sus pacientes complicados. Es decir, la diferencia en la mortalidad no estaba en que en los "mejores hospitales" los pacientes se complicaban menos, sino que en ellos estaban dados los recursos para sacarlos adelante pese al desarrollo de complicaciones. Observaciones similares han seguido demostrando la fuerte asociación entre volumen del hospital y mortalidad $^{43,44}$.

\section{Conclusiones}

Si volvemos a considerar que los resultados quirúrgicos interesan a muchos es fundamental, hoy, poder conocer y comparar éstos entre diferentes instituciones y en una misma institución en el transcurso del tiempo ${ }^{45-48}$. Sabemos que para ello debemos ajustar los resultados de acuerdo a los factores de riesgo y que, finalmente, están determinados por múltiples factores ${ }^{49,50}$. Están en juego factores del paciente, muchos de ellos no modificables. Otros dependen de la estructura del centro de que se trate; sus recursos y especialización de sus profesionales. También influyen los procesos de atención, que deben basarse en la mejor evidencia; éstos pueden revisarse periódicamente y traspasarse como buenas prácticas de un centro a otro. Este concepto hay ha llevado a hablar de "hospitales de alto valor", donde se observa una alta calidad a un bajo costo ${ }^{51}$.

\section{Referencias}

1. Greenberg CC, Kennedy GD. Advancing Quality Measurement to Include the Patient Perspective. Ann Surg 2014; 260: 10-2.

2. Donabedian A. Explorations in Quality Assessment and Monitoring The Definition and Quality and Approaches to Its Assessment. Vol 1, Ann Arbor, MI. Health Admin. Press, 1980.

3. Russell T. Safety and Quality Improvement in Surgical Practice. Ann Surg 2006; 244: 653-5.

4. Rikkers LF, Hoyt DB, Flum DR, Malangoni MA. Quality. The Key to Surgery's Future. Ann Surg 2014; 260: 567-76.

5. Dimick JB, Weeks WB, Karia RJ, Das S, Campbell DA. Who Pays for a Poor Surgical Quality? Building a Business Case for Quality Improvement. J Am Coll Surg 2006; 202: 933-7.

6. Clavien P-A. Targeting Quality in Surgery. Ann Surg 2013; 258: 659-68.

7. Martin RCG, Brennan MF, Jacques DP. Quality of Complication Reporting in the Surgical Literature. Ann Surg 2002; 235: 803-13.

8. Hutter MM, Rowell KS, Davaney LA, Sokal SM, Warshaw AL, Abbott WM, et al. Identification of Surgical 
Complications and Death: An Assessment of the Traditional Surgical Morbidity and Mortality Conference Compared with the American College of Surgeons-National Surgical Quality Improvement Program. J Am Coll Surg 2006; 203: 618-24.

9. Gore DC. National survey of surgical morbidity and mortality conferences. Am J Surg 2006; 191: 708-14.

10. Clavien PA, Strasberg SM. Severity Grading of Surgical Complications. Ann Surg 2009; 250: 197-8.

11. Clavien PA, Sanabria JR, Strasberg SM. Proposed classification of complications of surgery with examples of utility in cholecystectomy. Surgery 1992; 111: 518-26.

12. Dindo D, Demartines N, Clavien PA. Classification of Surgical Complications. A New Proposal With Evaluation in a Cohort of 6336 Patients and Results of a Survey. Ann Surg 2004; 240: 205-13.

13. Clavien PA, Barkun J, de Oliveira ML, Vauthey JN, Dindo D, Schulick RD, et al. The Clavien-Dindo Classification of Surgical Complications. Five-Year Experience. Ann Surg 2009; 250: 187-96.

14. Strasberg SM, Linehan DC, Hawkins WG. The Accordion Severity Grading System of Surgical Complications. Ann Surg 2009; 250: 177-86.

15. Daley J, Khuri SF, Henderson W, Hur K, Gibbs JO, Barbour G, et al. Risk Adjustment of the Postoperative Morbidity Rate for the Comparative Assessment of the Quality of Surgical Care: Results of the National Veterans Affairs Surgical Risk Study. J Am Coll Surg 1997; 185: 328-40.

16. Khuri SF, Daley J, Henderson W, Hur K, Demakis J, Aust JB, et al. The Department of Veterans Affarirs' NSQIP. The First National, Validated, Outcome-Based, Risk-Adjusted, and Peer-Controlled Program for the Measurement and Enhancement of Quality of Surgical Care. Ann Surg 1998; 228: 491-503.

17. Copeland GP, Jones D, Walters M. POSSUM: a scoring system for surgical audit. Br J Surg 1991; 78: 356-60.

18. Haga Y, Ikei S, Ogawa M. Estimation of Physiologic Ability and Surgical Stress (E-PASS) as a New Prediction Scoring System for Postoperative Morbidity and Mortality Following Elective Gastrointestinal Surgery. Surg Today 1999; 29: 219-25.

19. de Castro SMM, Houwert JT, Lagarde SM, Reitsma JB, Busch ORC, van Gulik TM, et al. Evaluation of POSSUM for Patients Undergoing Pancreatoduodenetomy. World J Surg 2009; 33: 1481-7.

20. Prytherch DR, Whiteley MS, Higgins B, Weaver PC, Prout WG, Powell SJ. POSSUM and Portsmouth POSSUM for predicting mortality. Br J Surg 1998; 85: 1217 20.

21. Mohil RS, Bhatnagar D, Bahadur L, Rajneesh, Dev DK,
Magan M: POSSUM and P-POSSUM for risk-adjusted audit of patients undergoing emergency laparotomy. $\mathrm{Br}$ J Surg 2004; 91: 500-3.

22. Haga Y, Ikei S, Wada Y, Takeuchi H, Sameshima H, Kimura O, et al. Evaluation of an Estimation of Physiologic Ability and Surgical Stress (E-PASS) Scoring System to Predict Postoperative Risk: A Multicenter Prospective Study. Surg Today 2001; 31: 569-74.

23. Banz VM, Studer P, Inderbitzin D, Candinas D. Validation of the Estimation of Physiologic Ability and Surgical Stress (E-PASS) Score in Liver Surgery. World J Surg 2009; 33: 1259-65.

24. Oka Y, Nishijima J, Oku K, Azuma T, Inada K, Miyazaki S, et al. Usefulnees of an Estimation of Physiologic Ability and Surgical Stress (E-PASS) Scoring System to Predict the Incidence of Postoperative Complications in Gastrointestinal Surgery. World J Surg 2005; 29: 102933.

25. Ingraham AM, Richards KE, Hall BL, Ko CY. Quality improvement in surgery: the American College of Surgeons National Surgical Quality Improvement Program approach. Adv Surg 2010; 44: 251-67.

26. Bilimoria KY, Liu Y, Paruch J, Zhou L, Kmiecik TE, Ko CY, et al. Development and Evaluation of the Universal ACS NSQIP Surgical Risk Calculator: A Decision Aide and Informed Consent Tool for Patients and Surgeons. J Am Coll Surg 2013; 217: 833-42.

27. Khuri SF. The NSQIP: A new frontier in surgery. Surgery 2005 ; 138: 837-43.

28. Lee JA, Morrison SL, Morris JN. Fatality from three common surgical conditions in teaching and non-teaching hospitals. Lancet 1957; 273: 785-91.

29. Luft HS, Bunker JP, Enthoven AC. Should operations be regionalized? The empirical relation between surgical volume and mortality. N Engl J Med 1979; 301: 1364-9.

30. Birkmeyer JD, Stuckel TA, Siewers AE, Goodney PP, Wennberg DE, Lucas FL. Surgeon Volume and Operative Mortality in the United States. N Engl J Surg 2003; 349: 2117-27.

31. Birkmeyer JD, Finlayson EVA, Birkmeyer CM. Volume standards for high-risk surgical procedures: Potential benefits of the Leapfrog initiative. Surgery 2001; 130: 415-22.

32. Finlayson EVA, Goodney PP, Birkmeyer JD. Hospital Volume and operative Mortality in Cancer Surgery. A National Study. Arch Surg 2003; 138: 721-5.

33. Birkmeyer JD, Siewers AE, Finlayson EVA, Stukel TA, Lucas FL, Batista I, et al. Hospital Volume and Surgical Mortality in the United States. N Engl J Med 2002; 346: 1128-37. 
34. Dudley RA, Johansen KL, Brand R, Rennie DJ, Milstein A. Selective Referral to High-Volume Hospitals. Estimating Potentially Avoidable Death. JAMA 2000; 283: 1159-66.

35. Hannan EL, Radzyner M, Rubin D, Dougherty J, Brennan MF. The influence of hospital and surgeon volume on in-hospital mortality for colectomy, gastrectomy, and lung lobectomy in patients with cancer. Surgery 2002; 131: 6-15.

36. Birkmeyer JD, Sun Y, Wong SL, Stuckel TA. Hospital Volume and Late Survival After Cancer Surgery. Ann Surg 2007; 245: 777-83.

37. Brennan MF, Radzyner M, Rubin DM. Outcome-More Than Just Operative Mortality. J Surg Oncol 2009; 99: 470-7.

38. Nathan H, Cameron JL, Choti MA, Schulick RD, Pawlick TM. The Volume-Outcomes Effects in Hepato-Pancreato-Biliary Surgery: Hospital Versus Surgeon Contributions and Specificity on the Ralationship. J Am Coll Surg 2009; 208: 528-38.

39. Ghaferi AA, Birkmeyer JD, Dimick JB. Complications, Failures to Rescue, and Mortality With Major Inpatient Surgery in Medicare Patients. Ann Surg 2009; 250: 1029 34.

40. Bilimoria KY, Phillips JD, Rock CE, Hayman A, Prystowsky JB, Bentrem DJ. Effect of Surgeon Training, Specialization, and Experience on Outcomes for Cancer Surgery: A Systematic Review of the Literature. Ann Surg Oncol 2009; 16: 1799-808.

41. Marathappu M, Gilbert BJ, El-Harasis MA, Nagendran M, McCulloch P, Duclos A, et al. The influence of Volume and Experience on Individual Surgical Performance: A Systematic Review. Ann Surg 2015; 261: 642-7.
42. Sheetz KH, Waits SA, Krell RW, Campbell DA, Englesbe MJ, Ghaferi AA. Improving Mortality Emergency Surgery in Older Patients Requires Focus on Complications Rescue. Ann Surg 2013; 258: 614-8.

43. Reames BN, Ghaferi AA, Birkmeyer JD, Dimick JB. Hospital Volume and Operative Mortality in the Modern Era. Ann Surg 2014; 260: 244-51.

44. Finks JF, Osborne NH, Birkmeyer JD. Trends in Hospital Volume and Operative Mortality for High-Risk Surgery. N Engl J Med 2011; 364: 2128-37.

45. Cooperberg MR, Birkmeyer JD, Litwin MS. Defining high quality health care. Urologic Oncology 2009; 27: 411-6.

46. Cohen ME, Ko CY, Bilimoria KY, Zhou L, Huffman K, Wang X, et al. Optimizing ACS NSQIP Modeling for Evaluation of Surgical Quality and Risk: Patient Risk Adjustement, Procedure Mix Adjustement, Shrinkage Adjustement, and Surgical Focus. J Am Coll Surg 2013; 217: 336-46.

47. Daley J, Henderson WG, Khuri SF. Risk-Adjusted Surgical Outcomes. Annu Rev Med 2001; 52: 275-87.

48. Flum DR, Pellegrini CA. The Business of Quality in Surgery. Ann Surg 2012; 255: 6-7.

49. Birkmeyer JD, Dimick JB, Birkmeyer NJO. Measuring the Quality of Surgical Care: Structure, Process, or Outcomes? J Am Coll Surg 2004; 198: 626-32.

50. Birkmeyer JD, Dimick JB. Understanding and Reducing Variation in Surgical Mortality. Annu Rev Med 2009; 60: 405-15.

51. Lawson EH, Zingmond DS, Stey AM, Hall BL, Ko CY. Measuring Risk-Adjusted Value Using Medicare and ACS-NSQIP. Is High-Quality, Low-Cost Surgical Care Achievable Everywhere? Ann Surg 2014; 260: 668-79. 